\title{
Landmark models to define the age- adjusted risk of developing stage 1 type 1 diabetes across childhood and adolescence
}

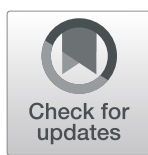

Verena Sophia Hoffmann ${ }^{1}$, Andreas Weiß ${ }^{1}$, Christiane Winkler ${ }^{1,2}$, Annette Knopff ${ }^{1}$, Manja Jolink ${ }^{1,2}$, Ezio Bonifacio $2,3,4^{*}+$ and Anette-G. Ziegler ${ }^{1,2,5^{*}+}$

\begin{abstract}
Background: Autoimmune diseases are often preceded by an asymptomatic autoantibody-positive phase. In type 1 diabetes, the detection of autoantibodies to pancreatic islet antigens in genetically at-risk children is prognostic for future clinical diabetes. Testing for islet autoantibodies is, therefore, performed in a range of clinical studies. Accurate risk estimates that consider the a priori genetic risk and other risk modifiers are an important component of screening. The age of an individual is an under-appreciated risk modifier. The aim of this study was to provide age-adjusted risk estimates for the development of autoantibodies across childhood in genetically at-risk children.

Methods: The prospective BABYDIAB and BABYDIET studies included 2441 children from birth who had a firstdegree relative with type 1 diabetes. Children were born between 1989 and 2006 and were regularly followed from birth for the development of islet autoantibodies and diabetes. A landmark analysis was performed to estimate the risk of islet autoantibodies at birth and at the age 3.5, 6.5 and 12.5 years. Exponential decay curves were fitted for the risk by the age of 20 years.

Results: The risk of islet autoantibodies by the age of 20 years was $8 \%, 4.6 \%, 2.6 \%$ and $0.9 \%$, at the landmark ages of birth, 3.5, 6.5 and 12.5 years, respectively. The short-term risks (within 6 years of follow-up) at these landmark ages were $5.3 \%, 2.9 \%, 1.8 \%$ and $1 \%$, respectively. The decline in autoantibody risk with age was modelled using a onephase exponential decay curve $(r=0.99)$ with a risk half-life of 3.7 years. This risk decay model was remarkably consistent when the outcome was defined as islet autoantibody-positive or multiple islet autoantibody-positive and when the study cohort was stratified by HLA risk genotype. A similar decay model was observed for coeliac diseaseassociated transglutaminase antibodies in the same cohort. Unlike the risk of developing islet autoantibodies, the rate of developing clinical diabetes in children who were islet autoantibody-positive did not decline with age.
\end{abstract}

Conclusion: The risk of developing autoantibodies drops exponentially with age in children with a first-degree relative with type 1 diabetes.

Keywords: Autoimmunity, Islet autoantibodies, Type 1 diabetes, Landmark risk

\footnotetext{
* Correspondence: ezio.bonifacio@tu-dresden.de; anette-g.ziegler@helmholtzmuenchen.de

Anette-G. Ziegler and Ezio Bonifacio are joint corresponding authors.

${ }^{2}$ Forschergruppe Diabetes e.V. at Helmholtz Zentrum München, German

Research Center for Environmental Health, Munich-Neuherberg, Germany

${ }^{1}$ Institute of Diabetes Research, Helmholtz Zentrum München, German

Research Center for Environmental Health, Ingolstaedter Landstr. 1, 85764

Munich-Neuherberg, Germany

Full list of author information is available at the end of the article
}

(c) The Author(s). 2019 Open Access This article is distributed under the terms of the Creative Commons Attribution 4.0 International License (http://creativecommons.org/licenses/by/4.0/), which permits unrestricted use, distribution, and

reproduction in any medium, provided you give appropriate credit to the original author(s) and the source, provide a link to the Creative Commons license, and indicate if changes were made. The Creative Commons Public Domain Dedication waiver (http://creativecommons.org/publicdomain/zero/1.0/) applies to the data made available in this article, unless otherwise stated. 


\section{Background}

The early asymptomatic phase of type 1 diabetes is detected by the presence of autoantibodies against pancreatic $\beta$ cell antigens such as insulin, glutamic acid decarboxylase (GAD), insulinoma-associated protein 2 (IA-2) and zinc transporter 8 (ZnT8) [1, 2]. Screening for islet autoantibodies is performed in people with a genetic predisposition for type 1 diabetes to investigate the natural history of the disease and when enrolling patients into interventional trials aimed at delaying the requirement for insulin replacement therapy [1, 3-9]. More recently, islet autoantibody screening has been piloted in the general population in Germany as part of routine healthcare to identify asymptomatic diabetes, known as stage 1 type 1 diabetes [10], and prevent diabetic ketoacidosis at the population level $[11,12]$.

An important aspect of offering tests for asymptomatic disease is the ability to determine accurate risk estimates for the group offered screening and to predict the prognosis of individuals with positive or negative test results. In cases where the a priori risk is increased, such as first-degree relatives of people with type 1 diabetes, it is important to provide them with accurate information on when to undergo testing and when their risk has changed. The risk of disease can change substantially with age. Although islet autoantibodies can appear throughout childhood and adolescence, they often appear early in life with a peak incidence period prior to 3 years of age [13-15]. Therefore, the risk of developing islet autoantibodies is likely to decline after this peak period of seroconversion. However, islet autoantibody risk estimates are rarely adjusted for the individual's age. The aim of this study was to define the age-adjusted risk of developing stage 1 type 1 diabetes across childhood and adolescence. Risks were determined in first-degree relatives of patients with type 1 diabetes who participated in two long-running German birth cohort studies, which have prospectively followed individuals from birth, with nearly 30 years of follow-up.

\section{Methods}

\section{Study population}

We used data from two German birth cohorts of individuals with a first-degree family history of type 1 diabetes born between 1989 and 2006 [7, 8]. The studies prospectively examined the natural history of islet autoimmunity and type 1 diabetes. The BABYDIAB study enrolled 1650 infants born to a mother or father with type 1 diabetes, and the BABYDIET study enrolled 791 infants who had a mother, father, or sibling with type 1 diabetes. A subgroup of 150 children with high-risk human leukocyte antigen (HLA) genotypes or two or more first-degree relatives with type 1 diabetes participated in the BABYDIET gluten intervention study (ClinicalTrials.gov NCT01115621) to investigate whether delaying exposure to gluten could reduce the risk of developing autoantibodies. The intervention failed to show an effect on islet autoantibody development and all participants continued with followup examinations according to the natural history protocol [8]. Children enrolled in the BABYDIAB or BABYDIET studies were scheduled for follow-up and venous blood collection at 9 months and 2 years of age, and every 3 years thereafter, whereas the 150 children participating in the dietary intervention were followed up with venous blood collection every 3 months until 3 years of age and yearly thereafter.

\section{Assessments of islet autoantibodies and diabetes}

Autoantibodies to insulin, GAD, IA-2 and ZnT8 were measured in samples taken at all scheduled visits and every 6 months in islet autoantibody-positive children. Transglutaminase autoantibodies were measured at all scheduled visits [16]. All autoantibody measurements were performed centrally by the Institute of Diabetes Research Munich using radiobinding assays and thresholds based on the upper 99th centile and Q-Q plots of results from control children as previously described [16-18]. Assay performance in international workshops is summarised in Additional file 1: Table S1.

Children were classified as islet autoantibody positive if they were positive for at least one islet autoantibody in at least two consecutive samples. The age of the first autoantibody-positive sample was considered the seroconversion age. Children were classified as multiple islet autoantibody-positive if, in addition to persisting islet autoantibody positivity, they tested positive for more than one islet autoantibody on at least one occasion. Genetic typing at HLA-DRB1, HLA-DQA1 and HLA$D Q B 1$ loci was performed as previously described [19].

Oral glucose tolerance tests were performed annually in islet autoantibody-positive children. Type 1 diabetes was diagnosed according to the American Diabetes Association Expert Committee criteria [20]. Families of children who dropped out of the study or refused to provide blood samples or perform oral glucose tolerance tests were regularly contacted by telephone and were asked if the child had developed diabetes.

\section{Statistical analyses}

To assess the time-dependent influence of age, cumulative islet autoantibody risks were calculated for children who remained islet autoantibody negative from birth or the respective landmark to the first islet autoantibodypositive state and to first multiple autoantibody-positive state. Cumulative risks were also calculated for progression to clinical diabetes in islet autoantibody-positive children. We determined landmark models [21] based on the cumulative incidence curves for the time from 
birth or from the respective landmark age to the first islet autoantibody-positive state, to the first multiple autoantibody-positive state and to clinical diabetes. The landmark ages were birth, 3.5, 6.5 and 12.5 years and were chosen at time points between scheduled visits to allow confirmation of positives prior to the landmark age. The follow-up time was set to 6 years (short-term risk), 12 years (mid-term risk) and 20 years (long-term risk).

Six-year dynamic prediction models were determined for all ages [22]. The prediction curves were produced using a Loess-based method [23]. Continuity correction, which accounted for the number at risk at each time point, was used to determine the $95 \%$ confidence intervals $(\mathrm{CI})$ as previously described [24].

Single-phase exponential decay functions were generated on the risk by age 20 years and by 6 years and 12 years of follow-up from the landmark ages of birth, 1.5, $3.5,6.5,9.5$ and 12.5 years to produce risk decay curves and equations and to calculate the risk half-life for each curve using the exponential decay curve fit function in
GraphPad Prism. The function was constricted to a plateau risk of $>0 \%$. The root mean square error was calculated as a measure of prediction error.

All analyses were performed separately for children with the HLA-DR3/4-DQ8 or HLA-DR4/4-DQ8 genotypes. Risks are given with 95\% CIs in parentheses. Analyses were performed using $\mathrm{R}$ version 3.4 .4 also using the package dynpred and GraphPad Prism version 8.0.1. (GraphPad Software, San Diego, CA, USA).

\section{Results}

Overall, 2441 children (1188 girls; 49\%) were enrolled at birth into the BABYDIAB and BABYDIET studies for prospective follow-up and were included in the present analyses. At the time of the analysis (December 2018), 164 children had developed at least one islet autoantibody, 132 had developed multiple islet autoantibodies and 115 had developed type 1 diabetes, including 10 children where type 1 diabetes was diagnosed without prior detection of islet autoantibodies.
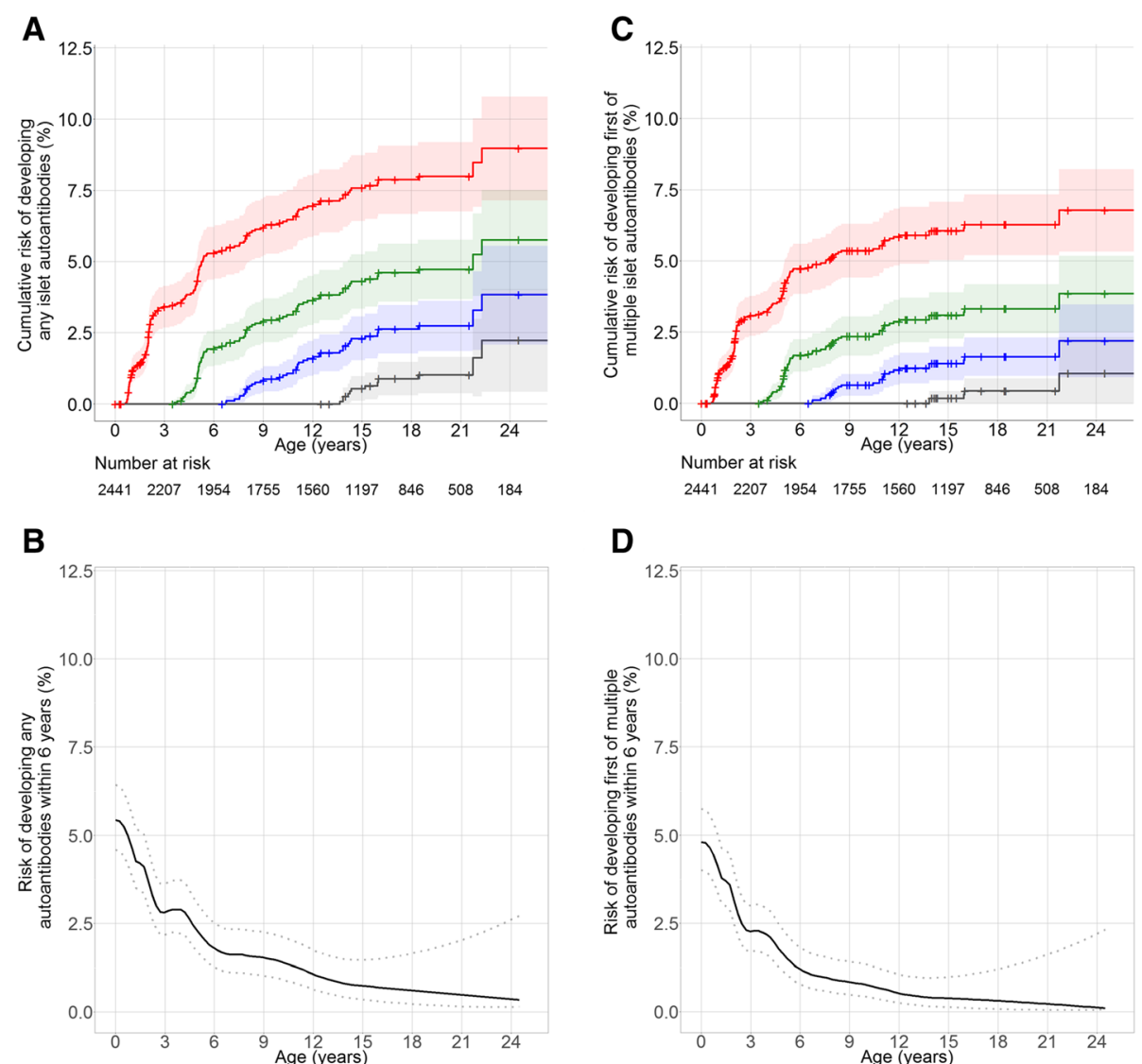

Fig. 1 Cumulative risks of developing islet autoantibodies. a, c Cumulative risks of developing any (a) or multiple islet autoantibodies (c) in the total cohort from birth (red), 3.5 years (green), 6.5 years (blue) and 12.5 years (grey) of age. b, $\mathbf{d}$ Dynamic predictions for the risks of developing any (b) or multiple (d) islet autoantibodies during the next 6 years of life 


\section{Landmark risks for islet autoantibodies}

Landmark models demonstrated decreasing short-term (6 years of follow-up), mid-term (12 years of follow-up) and long-term (20 years) risks of developing islet autoantibodies and multiple islet autoantibodies with increasing age of the children (Fig. 1, Table 1). The cumulative risks (95\% CI) of developing islet autoantibodies after 12 years of follow-up from the landmark ages of birth, 3.5, 6.5 and 12.5 years were $7.0 \%(5.9-8.0 \%), 4.4 \%$ (3.4-5.3\%), $2.7 \%$ $(1.9-3.6 \%)$ and $2.2 \%(0.4-4.0 \%)$, respectively (Fig. 1a); and the 12-year cumulative risks $(95 \% \mathrm{CI})$ for developing multiple islet autoantibodies were 5.8\% (4.8-6.8\%), 3.1\% (2.3$3.9 \%), 1.6 \%(1.0-2.3 \%)$ and $1.1 \%(0-2.3 \%)$, respectively (Fig. 1b; Table 1). The short-term (6 years) risk of developing islet autoantibodies decreased rapidly with increasing age (Fig. 1b, d).

Although risks were higher, similar relationships between landmark age and the risk of developing islet autoantibodies were observed when the analysis was restricted to children with the high-risk HLA-DR3-DR4DQ8 or DR4-DQ8/DR4-DQ8 genotypes (Fig. 2, Additional file 1: Table S2).

\section{Modelling the age-related autoantibody risk decline}

The decline in risk of islet autoantibodies with increasing age was remarkably consistent regardless of the outcome and duration of follow-up. Therefore, we sought to model the rate of decline using a curve fit function. A one-phase exponential decay function described the risk reduction with landmark age (RMSE = 0.155 for any islet autoantibodies and 0.074 for multiple islet autoantibodies; Fig. 3a; Additional file 1: Figure S1). The risk half-life was 3.7 years $(95 \% \mathrm{CI}$, 2.9-5.1 years) for developing any islet autoantibodies and 3.2 years (95\% CI, 2.6-4.0 years) for developing multiple islet autoantibodies by the age of 20 years. The risk of developing islet autoantibodies by the age of 20 years for any given age was described by the equation: risk at age $X=8.8 \times$ exp. $(-0.190 \times$ age $X)$, where age $X$ is the age at testing in the first-degree relative. The equation for multiple islet autoantibodies was a risk at age $X=7 \times \exp .(-0.219 \times$ age $X)$. For relatives with the high-risk HLA-DR3-DR4-DQ8 or DR4-DQ8/DR4-DQ8 genotypes, the exponential decay function yielded a risk half-life of 3.4 years $(95 \% \mathrm{CI}$,

Table 1 Landmark model of cumulative risks of developing islet autoantibodies and type 1 diabetes

\begin{tabular}{|c|c|c|c|c|}
\hline Outcome & Landmark age & Risk by 20 years of age & Risk after 6 years of follow-up & Risk after 12 years of follow-up \\
\hline \multirow[t]{6}{*}{ Any autoantibody } & From birth & $8.0 \%(6.8-9.2 \%)$ & $5.3 \%(4.4-6.2 \%)$ & $7.0 \%(5.9-8.0 \%)$ \\
\hline & From 1.5 years & $6.7 \%(5.5-7.8 \%)$ & $4.2 \%(3.3-5.0 \%)$ & $5.8 \%(4.8-6.9 \%)$ \\
\hline & From 3.5 years & $4.7 \%(3.7-5.8 \%)$ & $2.9 \%(2.2-3.7 \%)$ & $4.4 \%(3.4-5.3 \%)$ \\
\hline & From 6.5 years & $2.7 \%(1.9-3.6 \%)$ & $1.8 \%(1.1-2.4 \%)$ & $2.7 \%(1.9-3.6 \%)$ \\
\hline & From 9.5 years & $2.0 \%(1.2-2.8 \%)$ & $1.6 \%(0.9-2.3 \%)$ & $2.0 \%(1.2-2.8 \%)$ \\
\hline & From 12.5 years & $1.0 \%(0.4-1.7 \%)$ & $1.0 \%(0.4-1.7 \%)$ & $2.2 \%(0.4-4.0 \%)$ \\
\hline \multirow[t]{6}{*}{ Multiple autoantibodies } & From birth & $6.3 \%(5.2-7.3 \%)$ & $4.7 \%(3.8-5.6 \%)$ & $5.8 \%(4.8-6.8 \%)$ \\
\hline & From 1.5 years & $4.8 \%(3.9-5.8 \%)$ & $3.7 \%(2.9-4.4 \%)$ & $4.5 \%(3.6-5.4 \%)$ \\
\hline & From 3.5 years & $3.3 \%(2.5-4.2 \%)$ & $2.4 \%(1.7-3.0 \%)$ & $3.1 \%(2.3-3.9 \%)$ \\
\hline & From 6.5 years & $1.6 \%(1.0-2.3 \%)$ & $1.2 \%(0.7-1.8 \%)$ & $1.6 \%(1.0-2.3 \%)$ \\
\hline & From 9.5 years & $0.9 \%(0.4-1.4 \%)$ & $0.7 \%(0.2-1.1 \%)$ & $0.9 \%(0.4-1.4 \%)$ \\
\hline & From 12.5 years & $0.4 \%(0-0.9 \%)$ & $0.4 \%(0-0.9 \%)$ & $1.1 \%(0-2.3 \%)$ \\
\hline \multirow[t]{6}{*}{ Type 1 diabetes } & From birth & $6.2 \%(5.0-7.3 \%)$ & $1.4 \%(0.9-1.9 \%)$ & $3.7 \%(2.9-4.5 \%)$ \\
\hline & From 1.5 years & $5.9 \%(4.8-7.1 \%)$ & $1.6 \%(1.1-2.2 \%)$ & $4.2 \%(3.3-5.0 \%)$ \\
\hline & From 3.5 years & $5.4 \%(4.3-6.5 \%)$ & $1.8 \%(1.2-2.3 \%)$ & $4.2 \%(3.3-5.1 \%)$ \\
\hline & From 6.5 years & $4.7 \%(3.7-5.8 \%)$ & $2.4 \%(1.7-3.1 \%)$ & $4.5 \%(3.5-5.5 \%)$ \\
\hline & From 9.5 years & $3.7 \%(2.7-4.7 \%)$ & $2.5 \%(1.7-3.2 \%)$ & $3.7 \%(2.7-4.7 \%)$ \\
\hline & From 12.5 years & $2.4 \%(1.5-3.3 \%)$ & $2.1 \%(1.3-2.9 \%)$ & $2.8 \%(1.6-4.0 \%)$ \\
\hline \multirow[t]{5}{*}{ Type 1 diabetes, if islet antibody positive } & From 1.5 years & $83.6 \%(56.4-93.8 \%)$ & $45.6 \%(22.7-61.7 \%)$ & $54.3 \%(30.0-70.2 \%)$ \\
\hline & From 3.5 years & $84.3 \%$ (69.7-91.9\%) & $44.4 \%(30.4-55.6 \%)$ & $70.7 \%(56.2-80.4 \%)$ \\
\hline & From 6.5 years & $76.2 \%(62.1-85.0 \%)$ & $44.0 \%(32.2-53.8 \%)$ & $71.3 \%(57.8-80.5 \%)$ \\
\hline & From 9.5 years & $64.1 \%(47.9-75.3 \%)$ & $42.2 \%(29.0-53.0 \%)$ & $64.1 \%(47.9-75.3 \%)$ \\
\hline & From 12.5 years & $47.8 \%$ (31.3-60.4\%) & $42.0 \%$ (26.8-54.1\%) & $47.8 \%$ (31.3-60.4\%) \\
\hline
\end{tabular}



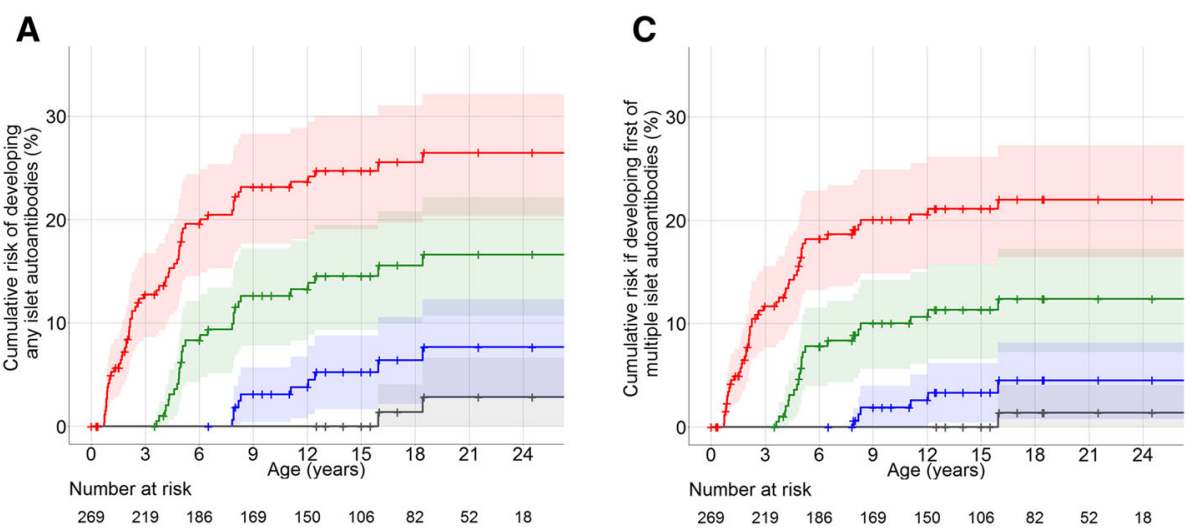

B
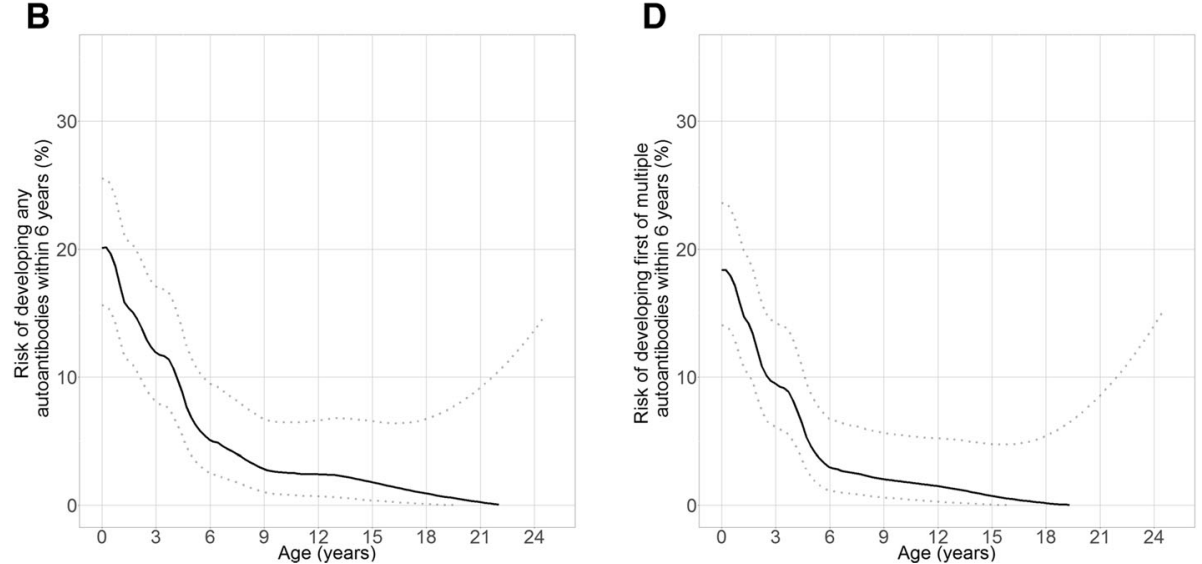

Fig. 2 Cumulative risks of developing islet autoantibodies in children with DR3/4-DQ8 or DR4-DQ8/DR4-DQ8 genotypes. a, c Cumulative risks of developing any (a) or multiple islet autoantibodies (c) in children with DR3/4-DQ8 or DR4-DQ8/DR4-DQ8 genotypes from birth (red), 3.5 years (green), 6.5 years (blue) and 12.5 years (grey) of age. $\mathbf{b}, \mathbf{d}$ Dynamic predictions for the risks of developing any (b) or multiple (d) islet autoantibodies during the next 6 years of life

2.3-7.0 years) for any islet autoantibody and 2.9 years (95\% CI, 2.2-4.2 years) for multiple islet autoantibodies by the age of 20 years (Fig. 3b).

Transglutaminase autoantibodies, which are associated with coeliac disease, were also measured in this cohort and provided us with an opportunity to test whether autoimmunity that often presents in childhood and with a partially known aetiology (exposure to gluten) displays a similar risk decay to islet autoimmunity (Additional file 1 : Figure S2, Additional file 1: Table S3). The risk declined after the age of 1.5 years and was described by an exponential decay function risk at age $X=9.1 \times$ exp. $(-0.290 \times$ age $X)$ ) with a half-life of 4.2 years ( $95 \%$ CI, 3.2-6.1 years). An exponential decay function was also observed for children with the genotype DR3/3, which conveys high transglutaminase autoantibody risk (Additional file 1: Figure S2c).

\section{Landmark risks for progression from islet autoantibody positivity to clinical type 1 diabetes}

Unlike the risk of developing islet autoantibodies, the 6year and 12-year risks of developing clinical diabetes in islet autoantibody-positive children (Fig. 4) and in multiple islet autoantibody-positive children (Additional file 1: Figure S3) did not change with increasing age. The cumulative risks $(95 \% \mathrm{CI})$ at 12 years of follow-up were 54.3\% (30.0-70.2\%), 70.7\% (56.2-80.4\%), 71.3\% (57.8$80.5 \%)$ and $47.8 \%(31.3-60.4 \%)$ in children who tested positive for islet autoantibodies at the ages of 1.5, 3.5, 6.5 and 12.5 years, respectively.

\section{Discussion}

In this study, we defined the influence of age on the risk of stage 1 type 1 diabetes among children with a firstdegree family history of type 1 diabetes. The risks decreased exponentially with a risk half-life of between 2.9 and 3.7 years for islet autoantibodies depending upon the a priori genetic risk and whether the outcome was the development of any islet autoantibody or multiple islet autoantibodies. However, the prognosis for clinical diabetes in children who had developed islet autoantibodies did not change with increasing age. 


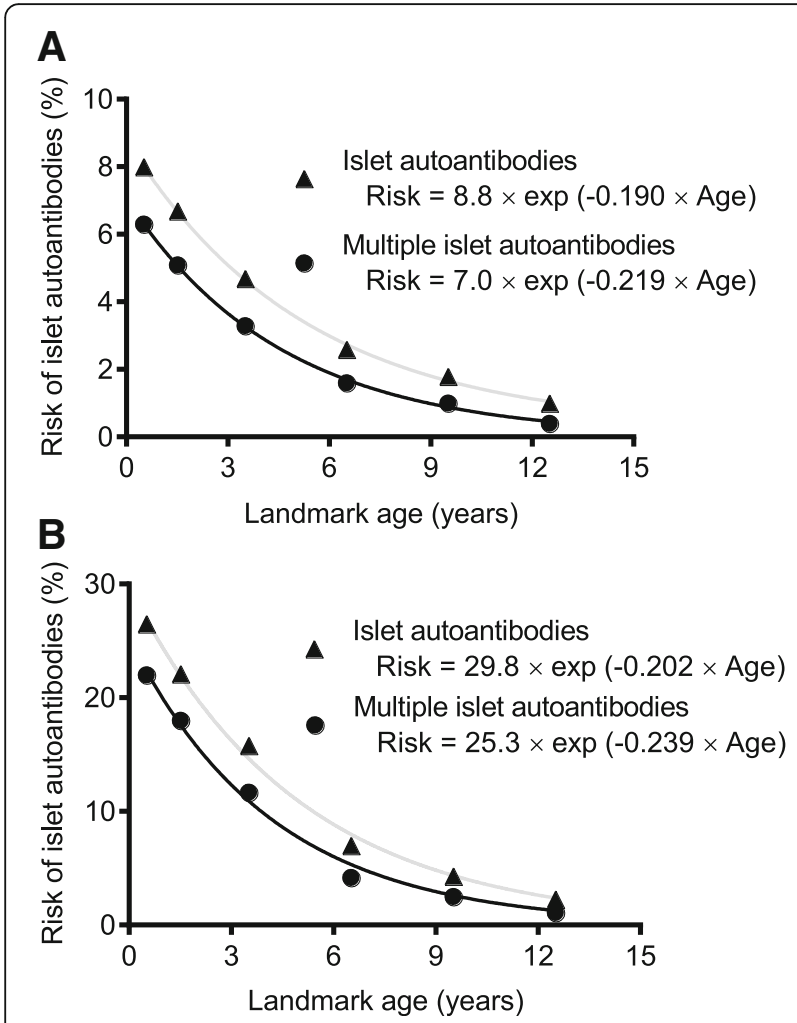

Fig. 3 One-phase exponential decay function for risk of developing islet autoantibodies by 20 years of age. a All children. $\mathbf{b}$ Children with the high-risk HLA DR3-DR4-DQ8 or DR4-DQ8/DR4-DQ8 genotypes

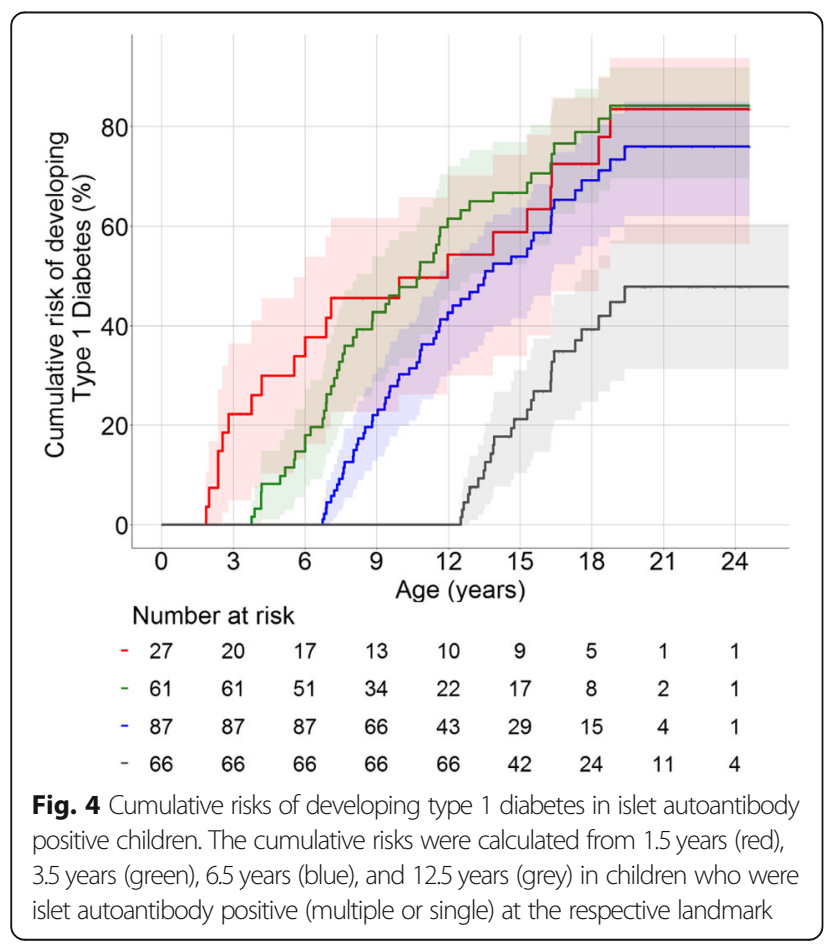

This is the first demonstration of a mathematical function describing the risk of autoimmunity at landmark ages. The relationship was consistently described by a single-phase exponential decay function for short-, midand long-term risks. These functions differed in terms of the overall risk from birth, but not the risk half-life. The description of risk throughout childhood was possible because of the uniquely long follow-up of this cohort. It is also possible that a single-phase decay function was observed because of the relatively homogeneous nature of the cohort, which comprised children who had a firstdegree relative with type 1 diabetes and who were followed from birth. It is known that the risk of developing islet autoantibodies differs according to which of the first-degree relatives is the proband $[25,26]$ and it is possible that the risk decay and the half-life may vary between children whose mother has type 1 diabetes and children whose father or sibling has type 1 diabetes. By restricting the analysis to children from affected families, we cannot assume that the risk decay is similar in children with non-familial genetic risk of type 1 diabetes. We and others have shown that autoimmunity to insulin often precedes autoimmunity to GAD [13-15], and the TEDDY study defined insulin-first and GAD-first endotypes [25]. It is, therefore, possible that the risk decay varies between each endotype and that the inclusion of other demographic and clinical factors, such as the child's sex and family history status, may be used to refine the risk decay models.

The findings of the study have practical value. Parents from affected families are often concerned about their child's risk of type 1 diabetes, which is $10-20$ times higher than that of a child in an unaffected family. Screening for islet autoantibodies can alleviate the concern if the result is negative, but a common question is whether a negative result implies that the child is no longer at high risk. Our findings indicate that the risk is reduced by 4 times by the time the children start school and by nearly 16 times once the children reach their teens, bringing it to a level similar to that of infants in the general population. Based on our findings, we suggest that screening of islet autoantibodies in children from affected families may be most beneficial if performed at the age of 2-3 years, which is shortly after the peak incidence of islet autoantibodies [13-15], again at $\sim 6$ years of age or entrance to elementary school, and in the early teens, after which time the risk of developing islet autoantibodies is not zero but no longer markedly elevated. The findings are also relevant to defining optimal screening ages for cohort and intervention studies.

It is possible to speculate on what the exponential risk decay implies for disease pathogenesis. While genetics markedly stratifies the magnitude of the risk of developing islet autoimmunity, the exponential decay was 
consistent in the overall cohort and the high-risk HLA subgroup. A single-phase exponential decay curve can be explained by a single initiation period and random development of islet autoantibodies thereafter. This scenario may imply that many of the genetic and nongenetic factors that determine islet autoimmunity act in the earliest years of life. We sought to provide further evidence for this possibility by using coeliac diseaseassociated autoimmunity where dietary gluten is known to be a major non-genetic aetiological risk factor. The findings for the development of transglutaminase autoantibodies mirrored those of islet autoimmunity with a later start and a longer half-life. As already discussed, a caveat is that the discriminatory power of multiple different risk decay curves is low in this cohort, and it is possible that there are different sets of risk factors for different endotypes.

The lack of a risk decay for prognosis in children who had developed islet autoantibodies also has practical value. This implies that the risk of becoming insulin dependent for an islet autoantibody-positive child remains constant, regardless of the child's age. Previous findings indicate that progression is faster in children who develop islet autoantibodies before the age of 3 years [1] and that progression is slower in autoantibodypositive adults than in autoantibody-positive children $[27,28]$. Therefore, the prognosis may be defined by a complex function with more than one phase that was not discernible in our cohort. A two-phase decay may explain the substantial proportion of patients developing the clinical disease in adulthood without a requirement for a second wave of islet autoimmunity at older ages. Pathogenetically, the relatively long progression half-life is consistent with scenarios of random development of diabetes after autoimmunity, or scenarios with one or few influencing factors that may occur at any age or multiple influencing factors occurring at different ages.

\section{Conclusion}

The development of islet autoimmunity in childhood follows an exponential decay model in affected families starting in the first year of life and with a risk half-life of $3-4$ years.

\section{Additional file}

Additional file 1: Table S1. Performance of the autoantibody assays used in the BABYDIAB and BABYDIET studies in the international Diabetes Autoantibody Standardisation Programme (DASP) and the Islet

Autoantibodies Standardisation Programme (IASP). Table S2. Landmark model of cumulative risks of developing islet autoantibodies and type 1 diabetes in children with the DR3/4-DQ8 or DR4-DQ8/DR4-DQ8 genotypes. Table S3. Landmark model of cumulative risks of developing transglutaminase autoantibodies. Figure S1. One-phase exponential decay functions of single and multiple islet autoantibodies for 6-year (black) and 12-year follow-up (blue). The 6-year exponential decay functions are $5.9 \times \exp .(-0.267 \times$ age $)$ and $5.3 \times$ exp. $(-0.241 \times$ age $)$ for single and multiple autoantibodies respectively. The 12-year exponential decay functions are $7.8 \times$ exp. $(-0.254 \times$ age $)$ and $6.6 \times$ exp. $(-0.272 \times$ age $)$ for single and multiple autoantibodies respectively. Figure S2. Cumulative Risks of developing transglutaminase autoantibodies (total population (A) and children with DR3/3 genotype (B)) from birth (red), from 3.5 years of age (green), from 6.5 years (blue) and from 12.5 years (grey), and one-phase exponential decay curves for total cohort and the high risk HLA DR3DR4-DQ8 or DR4-DQ8/DR4-DQ8 genotypes (C). Figure S3. Cumulative risks of developing type 1 diabetes in multiple islet autoantibody positive children. The cumulative risks were calculated from 1.5 years (red), 3.5 years (green), 6.5 years (blue) and 12.5 years (grey) in children who were multiple islet autoantibody positive at the respective landmark. (DOC $441 \mathrm{~kb}$ )

\section{Abbreviations}

Cl: Confidence interval; GAD: Glutamic acid decarboxylase; HLA: Human leukocyte antigen; IA-2: Insulinoma-associated protein 2; ZnT8: Zinc transporter 8

\section{Acknowledgements}

We thank all the families for participating in the BABYDIAB and BABYDIET studies. We also thank the primary care paediatricians and physicians for supporting the studies. We thank P. Achenbach for overseeing autoantibody measurements and M. Scholz, C. Matzke, C. Gezginci and L. Wendel for expert technical assistance.

\section{Authors' contributions}

VSH, AW, EB and AGZ were responsible for the study concept and design. $\mathrm{CW}, \mathrm{AK}$ and $\mathrm{MJ}$ collected the data and managed the database. VSH, AW, EB and AGZ analysed and interpreted the data, and drafted the report. All authors read and approved the final manuscript.

\section{Funding}

This work was funded by the German Federal Ministry of Education and Research to the German Center for Diabetes Research (DZD e.V.).

\section{Availability of data and materials}

The dataset analysed in this paper is available from the corresponding author on reasonable request, and with appropriate additional ethical approvals, where necessary.

\section{Ethics approval and consent to participate}

The BABYDIAB and the BABYDIET studies were approved by the ethics committee of Bavaria, Germany (Bayerische Landesärztekammer no. 95357 and Ludwig-Maximilians University no. 329/00, respectively) and were performed in accordance with the principles of the Declaration of Helsinki. Written informed consent was obtained from all participating families.

\section{Consent for publication}

Not applicable.

\section{Competing interests}

The authors declare that they have no competing interests.

\section{Author details}

${ }^{1}$ Institute of Diabetes Research, Helmholtz Zentrum München, German Research Center for Environmental Health, Ingolstaedter Landstr. 1, 85764 Munich-Neuherberg, Germany. ${ }^{2}$ Forschergruppe Diabetes e.V. at Helmholtz Zentrum München, German Research Center for Environmental Health, Munich-Neuherberg, Germany. ${ }^{3}$ Technische Universität Dresden, DFG Center for Regenerative Therapies Dresden, Fetscherstrasse 105, 01307 Dresden, Germany. ${ }^{4}$ Paul Langerhans Institute Dresden of the Helmholtz Center Munich at University Hospital Carl Gustav Carus and Faculty of Medicine, Dresden, TU, Germany. ${ }^{5}$ Forschergruppe Diabetes, Technical University Munich at Klinikum rechts der Isar, Munich, Germany. 
Received: 25 January 2019 Accepted: 4 June 2019

Published online: 09 July 2019

\section{References}

1. Ziegler AG, Rewers M, Simell O, Simell T, Lempainen J, Steck A, et al. Seroconversion to multiple islet autoantibodies and risk of progression to diabetes in children. JAMA. 2013;309(23):2473-9 https://doi.org/10.1001/ jama.2013.6285.

2. Insel RA, Dunne JL, Ziegler AG. General population screening for type 1 diabetes: has its time come? Curr Opin Endocrinol Diabetes Obes. 2015; 22(4):270-6 https://doi.org/10.1097/med.0000000000000173.

3. Greenbaum CJ, Speake C, Krischer J, Buckner J, Gottlieb PA, Schatz DA, et al. Strength in numbers: opportunities for enhancing the development of effective treatments for type 1 diabetes-the TrialNet experience. Diabetes. 2018;67(7):1216-25 https://doi.org/10.2337/db18-0065.

4. Nanto-Salonen K, Kupila A, Simell S, Siljander H, Salonsaari T, Hekkala A, et al. Nasal insulin to prevent type 1 diabetes in children with HLA genotypes and autoantibodies conferring increased risk of disease: a double-blind, randomised controlled trial. Lancet. 2008;372(9651):1746-55 https://doi.org/ 10.1016/s0140-6736(08)61309-4

5. Rewers M, Bugawan TL, Norris JM, Blair A, Beaty B, Hoffman M, et al. Newborn screening for HLA markers associated with IDDM: diabetes autoimmunity study in the young (DAISY). Diabetologia. 1996;39(7):807-12.

6. TEDDY Study Group. The Environmental Determinants of Diabetes in the Young (TEDDY) study: study design. Pediatric Diabetes. 2007;8(5):286-98. https://doi.org/10.1111/j.1399-5448.2007.00269.x.

7. Ziegler AG, Hillebrand B, Rabl W, Mayrhofer M, Mollenhauer U, Vordemann $J$, et al. On the appearance of islet associated autoimmunity in offspring of dabetes mothers: a prospective study from birth. Diabetologia. 1993;36(5): 402-8.

8. Hummel S, Pfluger M, Hummel M, Bonifacio E, Ziegler AG. Primary dietary intervention study to reduce the risk of islet autoimmunity in children at increased risk for type 1 diabetes: the BABYDIET study. Diabetes Care. 2011; 34(6):1301-5 https://doi.org/10.2337/dc10-2456.

9. Lundgren M, Sahlin Å, Svensson C, Carlsson A, Cedervall E, Jönsson B, et al. Reduced morbidity at diagnosis and improved glycemic control in children previously enrolled in DiPiS follow-up. Pediatr Diabetes. 2014;15(7):494-501 https://doi.org/10.1111/pedi.12151.

10. Insel RA, Dunne $J$, Atkinson MA, Chiang $U$, Dabelea D, Gottlieb PA, Greenbaum CJ, Herold KC, Krischer JP, Lernmark Å, Ratner RE, Rewers MJ, Schatz DA, Skyler JS, Sosenko JM, Ziegler AG. Staging presymptomatic type 1 diabetes: a scientific statement of JDRF, the Endocrine Society, and the American Diabetes Association. Diabetes Care. 2015;38(10):1964-74.

11. Raab J, Haupt F, Scholz M, Matzke C, Warncke K, Lange K, et al. Capillary blood islet autoantibody screening for identifying pre-type 1 diabetes in the general population: design and initial results of the Fr1da study. BMJ Open. 2016;6(5):e011144 https://doi.org/10.1136/bmjopen-2016-011144.

12. Kordonouri O, Lange $\mathrm{K}$, Boettcher I, Christoph J, Marquardt E, Tombois C, et al. New approach for detection of LDL-hypercholesterolemia in the pediatric population: the Fr1dolin-trial in Lower Saxony, Germany. Atherosclerosis. 2018;280:28085-91 https://doi.org/10.1016/j.atherosclerosis. 2018.11.011.

13. Ziegler AG, Bonifacio E. Age-related islet autoantibody incidence in offspring of patients with type 1 diabetes. Diabetologia. 2012;55(7):1937-43 https://doi.org/10.1007/s00125-012-2472-x.

14. Krischer JP, Lynch KF, Schatz DA, Ilonen J, Lernmark A, Hagopian WA, et al. The 6 year incidence of diabetes-associated autoantibodies in genetically at-risk children: the TEDDY study. Diabetologia. 2015;58(5):980-7 https://doi. org/10.1007/s00125-015-3514-y.

15. Parikka V, Nanto-Salonen $K$, Saarinen M, Simell T, llonen J, Hyoty H, et al. Early seroconversion and rapidly increasing autoantibody concentrations predict prepubertal manifestation of type 1 diabetes in children at genetic risk Diabetologia. 2012;55(7):1926-36 https://doi.org/10.1007/s00125-012-2523-3.

16. Hummel S, Hummel M, Banholzer J, Hanak D, Mollenhauer U, Bonifacio E, et al. Development of autoimmunity to transglutaminase $C$ in children of patients with type 1 diabetes: relationship to islet autoantibodies and infant feeding Diabetologia. 2007:50(2):390-4 https://doi.org/10.1007/s00125-006-0546-3.

17. Ziegler AG, Hummel M, Schenker M, Bonifacio E. Autoantibody appearance and risk for development of childhood diabetes in offspring of parents with type 1 diabetes: the 2-year analysis of the German BABYDIAB study. Diabetes. 1999;48(3):460-8.
18. Achenbach P, Lampasona V, Landherr U, Koczwara K, Krause S, Grallert H, et al. Autoantibodies to zinc transporter 8 and SLC30A8 genotype stratify type 1 diabetes risk. Diabetologia. 2009;52(9):1881-8 https://doi.org/10.1007/ s00125-009-1438-0.

19. Schenker M, Hummel M, Ferber K, Walter M, Keller E, Albert ED, et al. Early expression and high prevalence of islet autoantibodies for DR3/4 heterozygous and DR4/4 homozygous offspring of parents with type I diabetes: the German BABYDIAB study. Diabetologia. 1999;42(6):671-7 https://doi.org/10.1007/s001250051214.

20. Puavilai G, Chanprasertyotin S, Sriphrapradaeng A. Diagnostic criteria for diabetes mellitus and other categories of glucose intolerance: 1997 criteria by the Expert Committee on the Diagnosis and Classification of Diabetes Mellitus (ADA), 1998 WHO consultation criteria, and 1985 WHO criteria. World Health Organization. Diabetes Res Clin Pract. 1999;44(1):21-6. https:// doi.org/10.1016/S0168-8227(99)00008-X.

21. Putter $\mathrm{H}$, van Houwelingen $\mathrm{HC}$. Understanding landmarking and its relation with time-dependent Cox regression. Stat Biosci. 2017;9(2):489-503 https:// doi.org/10.1007/s12561-016-9157-9.

22. van Houwelingen $\mathrm{H}$, Putter $\mathrm{H}$. Dynamic prediction in clinical survival analysis. Boca Raton: CRC Press, Inc; 2011

23. Cleveland WS. Robust locally weighted regression and smoothing scatterplots. J Am Stat Assoc. 1979;74(368):829-36 https://doi.org/10.1080/ 01621459.1979.10481038.

24. Newcombe RG. Two-sided confidence intervals for the single proportion: comparison of seven methods. Stat Med. 1998;17(8):857-72.

25. Krischer JP, Lynch KF, Lernmark A, Hagopian WA, Rewers MJ, She JX, et al. Genetic and environmental interactions modify the risk of diabetes-related autoimmunity by 6 years of age: the TEDDY study. Diabetes Care. 2017; 40(9):1194-202 https://doi.org/10.2337/dc17-0238.

26. Bonifacio E, Pfluger M, Marienfeld S, Winkler C, Hummel M, Ziegler AG. Maternal type 1 diabetes reduces the risk of islet autoantibodies: relationships with birthweight and maternal $\mathrm{HbA}(1 \mathrm{c})$. Diabetologia. 2008; 51(7):1245-52 https://doi.org/10.1007/s00125-008-1022-Z.

27. Ziegler A-G, Bonifacio E. Group tB-BS. Age-related islet autoantibody incidence in offspring of patients with type 1 diabetes. Diabetologia. 2012; 55(7):1937-43 https://doi.org/10.1007/s00125-012-2472-x.

28. Bingley PJ, Gale EAM, Group TENDIT. Progression to type 1 diabetes in islet cell antibody-positive relatives in the European Nicotinamide Diabetes Intervention Trial: the role of additional immune, genetic and metabolic markers of risk. Diabetologia. 2006;49(5):881-90 https://doi.org/10.1007/ s00125-006-0160-4.

\section{Publisher's Note}

Springer Nature remains neutral with regard to jurisdictional claims in published maps and institutional affiliations.

Ready to submit your research? Choose BMC and benefit from

- fast, convenient online submission

- thorough peer review by experienced researchers in your field

- rapid publication on acceptance

- support for research data, including large and complex data types

- gold Open Access which fosters wider collaboration and increased citations

- maximum visibility for your research: over $100 \mathrm{M}$ website views per year

At $\mathrm{BMC}$, research is always in progress.

Learn more biomedcentral.com/submission 